\title{
Distributed Heuristic Adaptive Neural Networks With Variance Reduction in Switching Graphs
}

\section{Document Version}

Accepted author manuscript

Link to publication record in Manchester Research Explorer

\section{Citation for published version (APA):}

Liu, B., \& Ding, Z. (2019). Distributed Heuristic Adaptive Neural Networks With Variance Reduction in Switching Graphs. IEEE Transactions on Cybernetics. https://ieeexplore.ieee.org/document/8943129

\section{Published in:}

IEEE Transactions on Cybernetics

\section{Citing this paper}

Please note that where the full-text provided on Manchester Research Explorer is the Author Accepted Manuscript or Proof version this may differ from the final Published version. If citing, it is advised that you check and use the publisher's definitive version.

\section{General rights}

Copyright and moral rights for the publications made accessible in the Research Explorer are retained by the authors and/or other copyright owners and it is a condition of accessing publications that users recognise and abide by the legal requirements associated with these rights.

\section{Takedown policy}

If you believe that this document breaches copyright please refer to the University of Manchester's Takedown Procedures [http://man.ac.uk/04Y6Bo] or contact uml.scholarlycommunications@manchester.ac.uk providing relevant details, so we can investigate your claim.

\section{OPEN ACCESS}




\title{
Distributed Heuristic Adaptive Neural Networks With Variance Reduction in Switching Graphs
}

\author{
Bo Liu ${ }^{(1)}$ and Zhengtao Ding ${ }^{\circledR}$, Senior Member, IEEE
}

\begin{abstract}
This article proposes a distributed adaptive training method for neural networks in switching communication graphs to deal with the problems concerned with massive data or privacy-related data. First, the stochastic variance reduced gradient (SVRG) is used for the training of neural networks. Then, the authors propose a heuristic adaptive consensus algorithm for distributed training, which adaptively adjusts the weighted connectivity matrix based on the performance of each agent over the communication graph. Furthermore, it is proved that the proposed distributed heuristic adaptive neural networks ensure the convergence of all the agents to the optimum with a single communication among connected neighbors after every training step, which is also suitable for switching graphs. This theorem is verified by the simulation, which gives the results that fewer iterations are required for all agents to reach the optimum using the proposed heuristic adaptive consensus algorithm, and the SVRG can greatly decrease the fluctuations caused by the stochastic gradient and improve its performance with only a little extra computational cost.
\end{abstract}

Index Terms-Adaptive, consensus, distributed training, gradient descent, neural networks.

\section{INTRODUCTION}

W ITH the explosive growth of data volume and the increasing demand on privacy protection, distributed machine learning has become a hot research topic in the last decade for its great capacity in training with massive and privacy-related data, such as applications on big data [1], recommendation systems [2], and wireless sensor networks [3].

We consider the scenario that data samples are distributed stored on multiple agents over a graph, where the communication is only allowed among connected agents. However, data samples are unavailable to be exchanged among agents or collected centrally for some reason. First, the hardware or software limitation makes it infeasible for a single agent to process the massive data, for example, processors in the vehicle intelligent terminal [4]. Second, data samples are intrinsically stored on different devices or multisensors [5], where the data are generated or collected. Third, it is unavailable to share the data

Manuscript received November 21, 2018; revised July 17, 2019, October 17 , 2019; and November 15, 2019; accepted November 18, 2019. This work was supported by the Science and Technology Facilities Council through Newton Fund under Grant ST/N006852/1. This article was recommended by Associate Editor J. Cao. (Corresponding author: Zhengtao Ding.)

The authors are with the Department of Electrical and Electronic Engineering, University of Manchester, Manchester M13 9PL, U.K. (e-mail: bo.liu-2@manchester.ac.uk; zhengtao.ding@manchester.ac.uk)

Color versions of one or more of the figures in this article are available online at http://ieeexplore.iee.org.

Digital Object Identifier 10.1109/TCYB.2019.2956291 samples among agents or upload them to a centralized server out of privacy-related concerns, especially for those data about personal behavior [6] and medical use [7], [8]. However, we still expect that the model is trained based on the data from all agents, even though without the sharing of data samples. Therefore, parallel computation and distributed optimization methods are proposed to address this problem.

One significant advance in distributed optimization is the alternating direction method of multipliers (ADMM) method [9], which decomposes a large-scale optimization problem into many small subproblems, and then iteratively optimizes these subproblems in an alternating direction. ADMM is widely used in parallel optimization problems [10] such as distributed support vector machines (SVMs) [11], [12]. Another method for achieving distributed SVM is the cascade SVM, related theoretical and practical issues can be found in [13] and [14], the main idea of which is to share the support vectors among connected agents. The decentralized random vector functional link networks were proposed using a consensus-based method and ADMM in [15], which shows that these two methods exhibit comparable performance on the accuracy, while the consensus-based method has a faster convergence rate than ADMM.

Chen et al. [16] discussed the synchronization of multiple neural networks with time delay and proposed the eventtriggered control strategy to guarantee global exponential synchronization under some convenient conditions. The criterion of synchronization is feasible and does not require a Laplacian coupling matrix. Wang et al. [17] studied the coupled neural networks connected over both directed and undirected graphs and designed the appropriate adaptive laws and controllers to achieve synchronization in limited time. Ma and Bian [18] proposed a multiagent neuro-dynamic approach to address a class of distributed convex optimization problems with local constraints in continuous-time form, where all state solutions reach consensus asymptotically after each agent individually solves the objective function. The proposed method has low structural complexity and guarantees the existence and boundedness of the global state solution to the dynamical system.

With the combination of the consensus-based method [19] and neural networks, a distributed algorithm was designed in [20] to transform the centralized training into a decentralized form. However, each agent needs to communicate with its neighbors many times to achieve consensus, which is computationally expensive. Liu et al. [21] proposed a distributed neural networks framework based on the consensus 
algorithm, where all agents only need a single consensus step with their connected neighbors after every training update. Lian et al. [22] theoretically analyzed the centralized and decentralized algorithms on their convergence rate, which shows that the decentralized method has a faster convergence rate than its centralized counterpart on high latency or low bandwidth system and can bring asymptotically linear speed up when more agents are available.

In this article, we propose a distributed heuristic adaptive training algorithm in switching decentralized communication graphs, and a single consensus step is required among connected agents after each training iteration. Besides, the stochastic variance reduced gradient (SVRG) is used to optimize the neural networks on each agent to improve its convergence rate. After the analysis of the consensus-based distributed neural networks with a fixed weighted connectivity matrix, we design the heuristic adaptive consensus algorithm to adaptively adjust the weighted connectivity matrix, which speeds up the agents to reach the optimum. The theoretical analysis and simulation experiments give the result that the proposed heuristic adaptive consensus algorithm drives all agents to the optimal model faster, and the SVRG can greatly reduce the fluctuation caused by the stochastic gradient descent (SGD) and improve its convergence rate. The main contributions can be summarized as follows.

1) The heuristic adaptive consensus algorithm is proposed to adaptively adjust the weighted connectivity matrix for distributed training neural networks.

2) The proposed method is suitable for the switching communication graphs, which is robust to the failure of any agent.

\section{Stochastic Gradient Descent With Variance REDUCTION FOR NEURAL NETWORKS}

\section{A. Batch Gradient Descent}

Backpropagation is a popular method for the training of neural networks to minimize the empirical risk on a dataset, which consists of two iterative steps. That is, the computation of empirical risk and the optimization of model parameter using the gradient descent method

$$
\begin{aligned}
E(\mathcal{D}, \theta) & =\frac{1}{n} \sum_{i=1}^{n} Q\left(d_{i}, \theta\right) \\
Q\left(d_{i}, \theta\right) & =\frac{1}{2}\left(\hat{y}_{i}-y_{i}\right)^{2} \\
\hat{y}_{i} & =f\left(x_{i}, \theta\right)
\end{aligned}
$$

where $n$ is the number of data samples of $\mathcal{D}$, and $E(\mathcal{D}, \theta)$ and $Q\left(d_{i}, \theta\right)$ are the empirical risks over the model parameter $\theta$ on the dataset $\mathcal{D}$ and a sample $d_{i}=\left(x_{i}, y_{i}\right)$, respectively. $f$ is a function over the model parameter $\theta$, which maps $x_{i}$ to the $y_{i}$, with $\hat{y}_{i}$ being the estimated output.

Batch gradient descent [23] is a standard method to optimize the parameter $\theta$, the update rule of which is

$$
\begin{aligned}
\theta^{\prime} & =\theta-\eta \nabla E(\mathcal{D}, \theta) \\
\nabla E(\mathcal{D}, \theta) & =\frac{1}{n} \sum_{i=1}^{n} \nabla E\left(d_{i}, \theta\right)=\frac{1}{n} \sum_{i=1}^{n} \frac{\partial}{\partial \theta} Q\left(d_{i}, \theta\right)
\end{aligned}
$$

where $\theta^{\prime}$ is the trained model parameter of $\theta$, with $\eta$ representing the learning rate. $\nabla E\left(d_{i}, \theta\right)$ denotes the gradient on the sample $d_{i}$ over the model parameter $\theta$.

\section{B. Stochastic Variance Reduced Gradient}

We can find from (5) that $n$ derivatives need to be computed at each iteration for batch gradient descent, which could be computationally expensive and time consuming, especially, when the entire dataset is large. For this problem, SGD [24] is a good alternative method, which randomly selects a sample $d_{i}(i=1,2, \ldots, n)$ from the entire dataset to approximate the full gradient $\nabla E(\mathcal{D}, \theta)$. The update rule of SGD is

$$
\theta^{\prime}=\theta-\eta \nabla E\left(d_{i}, \theta\right)
$$

It is important to point out that the expectation $\mathbb{E}[\cdot]$ of the stochastic gradient $\nabla E\left(d_{i}, \theta\right)$ in (6) is the same as the full gradient in (4) because

$$
\mathbb{E}\left[\nabla E\left(d_{i}, \theta\right)\right]=\frac{1}{n} \sum_{i=1}^{n} \nabla E\left(d_{i}, \theta\right)=\mathbb{E}[\nabla E(\mathcal{D}, \theta)] .
$$

By estimating the full gradient using a single randomly selected sample at each iteration, SGD can greatly reduce the computational cost but incur the variance because of randomness. This kind of randomness may be helpful for the algorithm to escape from the local minimum, while too large variance also impairs its convergence rate, especially, when the learning rate is large. For this problem, the SVRG [25] method is used to reduce the variance, where an estimated parameter $\tilde{\theta}$ and its corresponding full gradient $\nabla E(\mathcal{D}, \tilde{\theta})$ are kept as a reference, and the gradient $v$ of SVRG is calculated by the following equation:

$$
\begin{aligned}
v & =\nabla E\left(d_{i}, \theta\right)-\nabla E\left(d_{i}, \tilde{\theta}\right)+\nabla E(\mathcal{D}, \tilde{\theta}) \\
\nabla E(\mathcal{D}, \tilde{\theta}) & =\frac{1}{n} \sum_{i=1}^{n} \nabla E\left(d_{i}, \tilde{\theta}\right)
\end{aligned}
$$

where $d_{i}$ represents a randomly selected sample, the $\nabla E\left(d_{i}, \theta\right)$ is the stochastic gradient of the random sample $d_{i}$ over the current model parameter $\theta, \nabla E\left(d_{i}, \tilde{\theta}\right)$ is the stochastic gradient of the random sample $d_{i}$ over the estimated model parameter $\tilde{\theta}$, and $\nabla E(\mathcal{D}, \tilde{\theta})$ is the full gradient on the entire dataset over the estimated model parameter $\tilde{\theta}$.

In (8), the item $\nabla E\left(d_{i}, \tilde{\theta}\right)$ is to reduce the variance caused by the randomness through the minus of $\nabla E\left(d_{i}, \theta\right)$ by $\nabla E\left(d_{i}, \tilde{\theta}\right)$, and the full gradient item $\nabla E(\mathcal{D}, \tilde{\theta})$ is to offset $\nabla E\left(d_{i}, \tilde{\theta}\right)$ because they are the same in expectation as explained in (7). We thus have that the gradient $v$ of SVRG is the same as the stochastic gradient in expectation, that is

$$
\begin{aligned}
\mathbb{E}[v] & =\mathbb{E}\left[\nabla E\left(d_{i}, \theta\right)-\nabla E\left(d_{i}, \tilde{\theta}\right)+\nabla E(\mathcal{D}, \tilde{\theta})\right] \\
& =\mathbb{E}\left[\nabla E\left(d_{i}, \theta\right)\right] .
\end{aligned}
$$

Notably, the full gradient does not need to be computed at every iteration but kept fixed over $m$ iterations, which only leads to less extra computational cost compared with SGD. More specifically, the computational complexity of batch gradient descent and SGD are $\mathcal{O}(n)$ and $\mathcal{O}(1)$ for each iteration, respectively, while that is $\mathcal{O}(2+[n / m])$ for SVRG. 


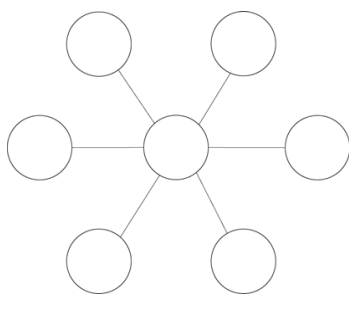

(a)

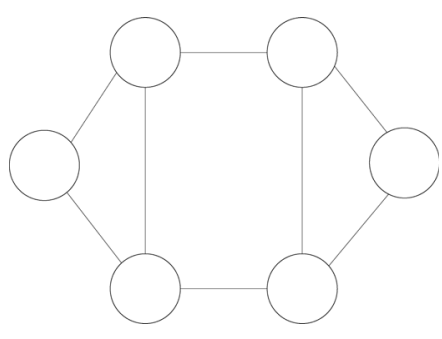

(b)
Fig. 1. (a) Master-slave topology. (b) Decentralized topology.

To analyze the convergence of (8), suppose that both $\tilde{\theta}$ and $\theta$ converge to the optimal $\theta^{*}$ after enough iterations, then $\nabla E(\mathcal{D}, \tilde{\theta}) \longrightarrow 0$ and $\nabla E\left(d_{i}, \tilde{\theta}\right) \longrightarrow \nabla E\left(d_{i}, \theta\right)$, therefore, $\nabla E\left(d_{i}, \theta\right)-\nabla E\left(d_{i}, \tilde{\theta}\right)+\nabla E(\mathcal{D}, \tilde{\theta}) \longrightarrow \nabla E\left(d_{i}, \theta\right)-$ $\nabla E\left(d_{i}, \tilde{\theta}\right) \longrightarrow 0$.

\section{Distributed Heuristic AdAptive NEURAL NETWORKS}

\section{A. Consensus Algorithm}

As shown in (5), we need to gather all data samples on a single agent to compute the full gradient $\nabla E(\mathcal{D}, \theta)$, which may be not available for those machine learning problems concerned with massive and privacy-related data. Therefore, parallel computation is introduced.

The master-slave topology in Fig. 1(a) is a typical centralized topology [26], where multiple slave agents are connected with a master agent. There are many exiting parallel algorithms for this communication topology, such as federated learning [27]. During each training iteration, all slave agents pull the latest model information from the master and compute the local gradients, which is then pushed to the master. After that, the master accumulates all the gradients from slave agents to update the model. Since the central agent is required to communicate with all other agents at every training step, there is a heavy communication load on the master in this centralized graph, especially for those systems with high latency and low bandwidth. More seriously, if the central agent fails for some reason, the whole distributed training framework would be inoperative.

In Fig. 1(b), the decentralized topology is designed to avert this problem, where no central node is required and each agent only shares the information with its neighbors. A decentralized communication topology is described by $\mathcal{G}=(\mathcal{V}, \mathcal{E})$, where $\mathcal{V}=\{1,2, \ldots, N\}$ and $\mathcal{E} \subset \mathcal{V} \times \mathcal{V}$ denote the set of agents and edges, respectively. An edge $(i, j) \in \mathcal{E}$ means that the communication between the $i$ th and $j$ th agents is allowed. Assuming there are $N$ agents over the topology, the connectivity among all agents formalizes a weighted connectivity matrix $W=\left[w_{i j}\right] \in \mathbb{R}^{N \times N}$, and it always satisfies that $w_{i j}>0$ when $(i, j) \in \mathcal{E}$ or $i=j$, otherwise, $w_{i j}=0$ [28]. Specifically, the communication strength between any two agents $i$ and $j$ is quantified by the value of the element $w_{i j}$, and $w_{i j}=0$ represents the disconnection of these two agents.

The undirected graph is considered in this article, the weighted connectivity matrix $W$ of which ought to satisfy some conditions to ensure the consensus of all agents, that is, $\sum_{j=1}^{N} w_{i j}=1 \forall i$ and $w_{i j}=w_{j i} \in[0,1) \forall(i, j)$. Assuming that the model parameter of each agent is denoted as a row vector $\theta_{i}$, the update rule of an agent with the consensus algorithm is

$$
\theta_{i}^{\prime}=\sum_{j=1}^{N} w_{i j} \theta_{j}
$$

where $\theta_{i}^{\prime}$ denotes the modified parameter vector of $\theta_{i}$ with a consensus step.

We then present the update rule for all agents using the consensus algorithm with a single step as

$$
\boldsymbol{\theta}^{\prime}=\mathcal{C}(\boldsymbol{\theta}, W)=W \boldsymbol{\theta}
$$

where $\boldsymbol{\theta}=\left[\theta_{1}, \theta_{2} \cdots \theta_{N}\right]^{T}$ is the model parameter matrix of all agents, with $\theta_{k}$ being the $k$ th row of $\boldsymbol{\theta} . \boldsymbol{\theta}^{\prime}$ is the updated parameter matrix after the consensus procedure $\mathcal{C}$.

The consensus algorithm draws the model parameter of all agents to approach their average with only local communication by the repetitive computation of (12) until good enough accuracy is reached. Practically, the number of repetitions influences the precision of the estimated average and consensus accuracy [29]. For a given decentralized graph topology, the weighted connectivity matrix $W$ depends on the chosen consensus strategy, which also has considerable influence on the rate of all agents to achieve consensus. We take the Laplacian method [30] as the consensus strategy in this article because of its fast convergence rate.

\section{B. Distributed Neural Networks}

Assume there are $N$ subdatasets distributed stored on $N$ agents over a graph, and the neural networks on all agents have the same initialization. After every training step, we compute the average of all agents' model parameters $\left(\theta_{1}, \theta_{1} \cdots \theta_{N}\right)$, which is subsequently sent to every agent as the new model parameter. Following this update rule, the distributed neural networks have similar performance as the neural network trained on the entire dataset, when the batch gradient descent is used to optimize the model parameter.

In the distributed training case, the empirical risk on the entire dataset is decomposed into many small empirical risks on different subdatasets

$$
\begin{aligned}
E\left({ }^{k} \mathcal{D}, \theta_{k}\right) & =\frac{1}{n_{k}} \sum_{i=1}^{n_{k}} Q\left({ }^{k} d_{i}, \theta_{k}\right) \\
\nabla E\left({ }^{k} \mathcal{D}, \theta_{k}\right) & =\frac{1}{n_{k}} \sum_{i=1}^{n_{k}} \frac{\partial}{\partial\left(\theta_{k}\right)} Q\left({ }^{k} d_{i}, \theta_{k}\right)
\end{aligned}
$$

where $\theta_{k}$ and ${ }^{k} \mathcal{D}$ denote the model parameter and subdataset of agent $k$, respectively. $n_{k}$ is the number of samples of ${ }^{k} \mathcal{D}$, with ${ }^{k} d_{i}$ being a data sample.

Combining (13) and (14) with (1) and (5), it is found that the empirical risk and gradient on the entire dataset is obtained by averaging them on subdatasets of all agents. 
This lead to

$$
\begin{aligned}
E(\mathcal{D}, \theta) & =\sum_{k=1}^{N} \frac{n_{k}}{n} \frac{1}{n_{k}} Q\left({ }^{k} d_{i}, \theta_{k}\right) \\
& =\sum_{k=1}^{N} \frac{n_{k}}{n} E\left({ }^{k} \mathcal{D}, \theta_{k}\right) \\
\nabla E(\mathcal{D}, \theta) & =\sum_{k=1}^{N} \frac{n_{k}}{n} \frac{1}{n_{k}} \frac{\partial}{\partial\left(\theta_{k}\right)} Q\left({ }^{k} d_{i}, \theta_{k}\right) \\
& =\sum_{k=1}^{N} \frac{n_{k}}{n} \nabla E\left({ }^{k} \mathcal{D}, \theta_{k}\right)
\end{aligned}
$$

where $N$ is the number of agents in the communication graph.

It is easy to find from (16) that the distributed training neural networks generate an identical gradient $\nabla E(\mathcal{D}, \theta)$ as the neural network trained on the entire dataset, by averaging the gradients $\nabla E\left({ }^{k} \mathcal{D}, \theta_{k}\right)$ from all agents with respect to the sizes of their subdatasets.

Then, we obtain the update procedures for the consensusbased distributed neural networks in a decentralized communication topology with enough consensus steps, that is

$$
\begin{aligned}
\nabla E^{\prime}(\boldsymbol{\theta}) & =\mathcal{C}(\nabla E(\boldsymbol{\theta}), W) \\
\theta_{k}^{\prime \prime} & =\theta_{k}-\eta \nabla E^{\prime}\left({ }^{k} \mathcal{D}, \theta_{k}\right)
\end{aligned}
$$

where $\nabla E^{\prime}(\boldsymbol{\theta})=\left[\nabla E^{\prime}\left({ }^{1} \mathcal{D}, \theta_{1}\right), \nabla E^{\prime}\left({ }^{2} \mathcal{D}, \theta_{2}\right) \cdots \nabla E^{\prime}\left({ }^{N} \mathcal{D}, \theta_{N}\right)\right]^{T}$ is the updated gradient matrix of $\nabla E(\boldsymbol{\theta})$ after the consensus procedure $\mathcal{C}$ with enough steps. $\theta_{k}^{\prime \prime}$ is the updated model parameter using the gradient descent method.

It is notable that the exchange of (17) and (18) does not influence the above analysis, and removes the condition that all agents have the same initialization. In this update criteria for distributed training, we first update the local model parameter $\theta_{k}$ with the gradient $\nabla E\left({ }^{k} \mathcal{D}, \theta_{k}\right)$, and then average all agents' model parameters with the consensus algorithm. The modified update rule is

$$
\begin{aligned}
\theta_{k}^{\prime} & =\theta_{k}-\eta \nabla E\left({ }^{k} \mathcal{D}, \theta_{k}\right) \\
\boldsymbol{\theta}^{\prime \prime} & =\mathcal{C}\left(\boldsymbol{\theta}^{\prime}, W\right)
\end{aligned}
$$

where $\theta_{k}^{\prime}$ denotes the trained model parameter of $\theta_{k}$ using the gradient descent, while $\boldsymbol{\theta}^{\prime \prime}$ represents the modified model parameter matrix of $\boldsymbol{\theta}^{\prime}$ with the consensus algorithm.

Same as (12), repetitive computing is required for (17) and (20) to reach consensus after each training iteration, which is computationally expensive. To alleviate the computation cost, we design the consensus-based distributed training neural networks, which requires a single consensus communication at every training step. This greatly reduces the communication cost caused by the consensus process. In the following sections, it is demonstrated by the proof and simulation that the consensus-based distributed training method guarantees the convergence of all agents to the optimum.

\section{Heuristic Adaptive Consensus Algorithm}

As explained above, the weighted connectivity matrix $W$ of the consensus algorithm is fixed throughout the training process, which means that an agent's influence on its neighbors is constant and only depends on the graph topology and the consensus strategy. This results in that all agents would approach each other equally during the consensus process, no matter how good or bad an agent's performance is.

A more reasonable idea is that the agent with better performance should exhibit more influence on its neighbors, that is, all the agents should approach a larger step to its better neighbors. Here, the root-mean-squared-error (RMSE) is applied to assess the performances of the agents. The straightforward idea is that the smaller the error of an agent is, the larger value it should have in the weighted connectivity matrix $W$. For a communication topology, we define a connectivity matrix $A=\left[a_{i j}\right] \in \mathbb{R}^{N \times N}$, where $a_{i j}=1$ if the edge $(i, j) \in \mathcal{E}$ or $i=j$; otherwise, $a_{i j}=0$. The weight matrix $C=\left[c_{i j}\right] \in \mathbb{R}^{N \times N}$ in terms of the RMSE $=\sqrt{E\left({ }^{i} \mathcal{D}, \theta_{i}\right)}$ of all agents is given by

$$
\begin{aligned}
c_{i j} & =\frac{a_{i j} e_{j}}{\sum_{j=1}^{N} a_{i j} e_{j}} \\
e_{i} & =\frac{1}{\sqrt{E\left({ }^{i} \mathcal{D}, \theta_{i}\right)}}
\end{aligned}
$$

where $e_{i}$ represents the reciprocal of the RMSE of agent $i$.

However, if the weight matrix $C$ directly depends on their performance, all agents will immediately converge to the currently better agent. This may lose the agents' search capability and impair its robustness. For example, in the situation that the currently best agent is in a local minimum, all other agents are more likely to be attracted into this basin of attraction with the local minimum based on the weight matrix $C$. The idea from the particle swarm optimization (PSO) [31], [32] is therefore introduced to design the weighted connectivity matrix $W$, where an agent with the smaller error only leads to the bigger possibility of obtaining a large value in the weighted connectivity matrix. Based on this, we can obtain the weighted connectivity matrix $W$ by the combination of a bounded random matrix $B=\left[b_{i j}\right] \in \mathbb{R}^{N \times N}$ and the weight matrix $C$

$$
\begin{aligned}
w_{i j} & =b_{i j} c_{i j} \\
b_{i j} & =\lambda \alpha+(1-\lambda)
\end{aligned}
$$

where $\alpha \in[0,1]$ is a random number, and $\lambda \in[0,1]$ is a user-defined parameter to determine the bound of the random matrix $B$.

In this way, $w_{i j} \in\left[(1-\lambda) b_{i j}, b_{i j}\right]$ is a random number defined by $b_{i j}$. Due to the fact that the better the performance of an agent is, the larger $b_{i j}$ is, the interval $\left[(1-\lambda) b_{i j}, b_{i j}\right]$, as well as the expectation of $w_{i j}$, thus adaptively changes according to the performances of agent $i$ and its directly connected neighbors. Even though that $w_{i j}$ is a random number within this interval, all agents will approach larger steps to their better neighbors in the long run and reserve the searching ability in the domain based on all agents by the randomness.

It is notable that the sum of any rows of the matrix $W$ obtained from (23) may be smaller than 1, that is, $\sum_{j=1}^{N} w_{i j}=$ $\sum_{j=1}^{N} b_{i j} c_{i j} \leq 1$ because $b_{i j} \leq 1$ and $\sum_{j=1}^{N} c_{i j}=1$, and this will result in divergence. To satisfy the requirement that $\sum_{j=1}^{N} w_{i j}=1 \forall i$, we define another weight parameter $\beta_{i}$, which 
is allocated to the historical best model parameter of agent $i$ up to the current iteration. Then, we obtain the new adaptive update rule for $\theta_{i}^{\prime}$

$$
\begin{aligned}
& \beta_{i}=1-\sum_{j=1}^{N} w_{i j} \\
& \theta_{i}^{\prime \prime}=\sum_{j=1}^{N} w_{i j} \theta_{j}^{\prime}+\beta_{i} \theta_{i}^{\prime *}
\end{aligned}
$$

where $\theta_{i}^{\prime \prime}$ denotes the optimized model parameter of $\theta_{i}^{\prime}$ using the heuristic adaptive consensus algorithm, with $\theta_{i}^{\prime *}$ being the historical best model parameter of agent $i$.

This heuristic adaptive consensus algorithm is more efficient and robust, compared with the consensus algorithm with a fixed weighted connectivity matrix. First, the value of the weight matrix $C$ depends on the performance of all agents, which means that each agent will adaptively approach a larger step to its better neighbors. Second, the introducing of the bounded random matrix $B$ keeps the agents' random searching ability during the consensus process, where the tunable parameter $\lambda$ can adjust the agents' local and global searching ability by defining the bound of matrix $B$. Third, the consideration of the historical best model parameter $\theta_{i}^{\prime *}$, to some extent, avoids the agent getting worse.

\section{Convergence Analysis}

In this section, it will be proved that, even though with a single consensus communication at every training step, the proposed distributed heuristic adaptive neural networks ensure all agents converge to the optimum. This also holds in the switching graphs and the situation that any new agent quits or participates in the communication graph.

Assumption 1: The training process $\mathcal{T}$ based on any dataset ${ }^{i} \mathcal{D}$ from the entire dataset $\mathcal{D}$ would lead to a contraction of the empirical risk, that is

$$
E\left(\mathcal{D}, \theta_{i}^{\prime}\right)=E\left(\mathcal{D}, \mathcal{T}\left({ }^{i} \mathcal{D}, \theta_{i}\right)\right) \leq \mu E\left(\mathcal{D}, \theta_{i}\right), 0<\mu<1
$$

where $E\left(\mathcal{D}, \theta_{i}\right.$, ) is the empirical risk of the $i$ th neural network with parameter $\theta_{i}$ over the entire dataset, and $\theta_{i}^{\prime}$ represents the updated $\theta_{i}$ by the training process $\mathcal{T}$ with dataset ${ }^{i} \mathcal{D}$. For simplicity, $E\left({ }^{i} \mathcal{D}, \theta_{i}\right)$ and $E\left({ }^{i} \mathcal{D}, \theta_{i}^{\prime}\right)$ are aliased as $E_{i}$ and $E_{i}^{\prime}$, respectively.

Assumption 2: $E(\mathcal{D}, \theta)$ is a convex function about $\theta$, with $\theta^{*}$ and $E^{*}$ being the optimal solution and the minimal empirical risk, respectively.

As shown in (26), $\theta_{i}^{\prime \prime}$ is the updated model parameter of $\theta_{i}^{\prime}$ using the heuristic adaptive consensus algorithm, the corresponding empirical risk is

$$
E\left(\theta_{i}^{\prime \prime}\right)=E\left(\sum_{j=1}^{N} w_{i j} \theta_{j}^{\prime}+\beta_{i} \theta_{i}^{* *}\right), i=(1,2, \ldots, N)
$$

where $E\left(\theta_{i}^{\prime \prime}\right)$ represents the empirical risk over the model parameter $\theta_{i}^{\prime \prime}$, which is aliased as $E_{i}^{\prime \prime}$.

Proposition 1: Based on the condition that the weighted connectivity matrix satisfies $w_{i j} \in[0,1)$ and $\sum_{j=1}^{N} w_{i j}+$ $\beta_{i}=1(i, j=1,2, \ldots, N)$, we have

$$
\left\|\left(E_{1}^{\prime \prime}, E_{2}^{\prime \prime}, \ldots, E_{N}^{\prime \prime}\right)\right\|_{\infty} \leq\left\|\left(E_{1}^{\prime}, E_{2}^{\prime}, \ldots, E_{N}^{\prime}\right)\right\|_{\infty}
$$

where $\|\cdot\|_{\infty}$ denotes the max-norm, the "=" holds when $E_{i}^{\prime}=$ $E_{j}^{\prime} \forall i, j$. Here, we define $\mathcal{F}^{\prime}=\left(E_{1}^{\prime}, E_{2}^{\prime}, \ldots, E_{N}^{\prime}\right)$ and $\mathcal{F}^{\prime \prime}=$ $\left(E_{1}^{\prime \prime}, E_{2}^{\prime \prime}, \ldots, E_{N}^{\prime \prime}\right)$.

Proof:

$$
\begin{aligned}
& \left\|\left(E_{1}^{\prime \prime}, E_{2}^{\prime \prime}, \ldots, E_{N}^{\prime \prime}\right)\right\|_{\infty} \\
& \quad=\max _{k}\left\|E_{k}^{\prime \prime}\right\| \\
& =\max _{k}\left\|E\left(\sum_{j=1}^{N} w_{k j} \theta_{j}^{\prime}+\beta_{k} \theta_{k}^{\prime *}\right)\right\| \\
& \leq \max _{k}\left\|E\left(\sum_{j=1, j \neq k}^{N} w_{k j} \theta_{j}^{\prime}+\left(w_{k k}+\beta_{k}\right) \theta_{k}^{\prime *}\right)\right\| \\
& \leq \max _{k}\left\|\sum_{j=1, j \neq k}^{N} w_{k j} E\left(\theta_{j}^{\prime}\right)+\left(w_{k k}+\beta_{k}\right) E\left(\theta_{k}^{\prime}\right)\right\| \\
& \leq \max _{k}\left\|E\left(\theta_{k}^{\prime}\right)\right\| \\
& =\left\|\left(E_{1}^{\prime}, E_{2}^{\prime}, \ldots, E_{N}^{\prime}\right)\right\|_{\infty} .
\end{aligned}
$$

Remark 1: The switching of graph topology and the joining or leaving of a new agent change the value and dimension of the connectivity matrix $A$, respectively. However, these changes would only influence the value or dimension of the weighted connectivity matrix $W$, which still meets the condition that $\sum_{j=1}^{N} w_{i j}+\beta_{i}=1(i=1,2, \ldots, N)$. Therefore, Proposition 1 still holds in switching communication graphs and the situation that any new agent quits or participates in the graph.

Theorem 1: Under Assumptions 1 and 2, all $E_{k}$ converge to the minimum $E^{*}$ in both fixed and switching graphs, despite their initial statues for the proposed distributed heuristic adaptive neural networks.

Proof: We define the maximal gap between the empirical risks of all agents $\mathcal{F}=\left(E_{1}, E_{2}, \ldots, E_{N}\right)$ and the minimum $E^{*}$ as $R(\mathcal{F})$, which is given by

$$
\begin{aligned}
R(\mathcal{F}) & =\max _{k}\left(\left\|E_{k}\right\|-\left\|E^{*}\right\|\right) \\
& =\left\|\left(E_{1}, E_{2}, \ldots, E_{N}\right)\right\|_{\infty}-\left\|E^{*}\right\|
\end{aligned}
$$

where it always holds that $\left\|E^{*}\right\| \leq\left\|E_{k}\right\|$.

For both the fixed graph and the switching graphs cases, by Proposition 1 to (31), we have

$$
\begin{aligned}
R\left(\mathcal{F}^{\prime \prime}\right) & =\max _{k}\left(\left\|E_{k}^{\prime \prime}\right\|-\left\|E^{*}\right\|\right) \\
& =\left\|\left(E_{1}^{\prime \prime}, E_{2}^{\prime \prime}, \ldots, E_{N}^{\prime \prime}\right)\right\|_{\infty}-\left\|E^{*}\right\| \\
& \leq\left\|\left(E_{1}^{\prime}, E_{2}^{\prime}, \ldots, E_{N}^{\prime}\right)\right\|_{\infty}-\left\|E^{*}\right\| \\
& =R\left(\mathcal{F}^{\prime}\right) .
\end{aligned}
$$

Based on (27), it is obtained

$$
\begin{aligned}
R\left(\mathcal{F}^{\prime}\right) & =\max _{k}\left(\left\|E_{k}^{\prime}\right\|-\left\|E^{*}\right\|\right) \\
& \leq \mu\left\|E_{k}\right\|-\left\|E^{*}\right\|
\end{aligned}
$$




$$
\begin{aligned}
& <\mu\left(\left\|\left(E_{1}, E_{2}, \ldots, E_{N}\right)\right\|_{\infty}-\left\|E^{*}\right\|\right) \\
& <R(\mathcal{F})
\end{aligned}
$$

where $0<\mu<1$. Therefore, we can obtain

$$
R\left(\mathcal{F}^{\prime \prime}\right) \leq R\left(\mathcal{F}^{\prime}\right)<R(\mathcal{F})
$$

Equation (34) implies that the maximal gap $R(\mathcal{F})$ keeps descending and all agents' empirical risks $\left(E_{1}, E_{2}, \ldots, E_{N}\right)$ will approach the minimum $E^{*}$, as the distributed training process carries on.

Remark 2: Assumption 1 is a common concept for neural network training problem, where a single or several training steps usually leads to a better model.

Remark 3: Assumption 2 is generally used to prove the convergence of the machine learning problems [33], [34]. The convexity of the empirical risk guarantees the decline of the largest empirical risk among all agents with the consensus process. This idea may also be suitable for the nonconvex neural networks optimization problem, which is validated in the numerical simulation.

\section{Simulation And Discussion}

There is a two-phase update process for the distributed heuristic adaptive neural networks, which consists of local training and global consensus. During the first phase of local training, each neural network (agent) trains the model parameter on its local subdatasets simultaneously, where the SVRG is used to reduce the variance introduced by SGD and improve its convergence rate. Then, all neural networks share their model parameters among connected neighbors to update the models in the second phase of global consensus, where the heuristic adaptive consensus algorithm is used to adaptively adjust the weighted connectivity matrix to speed up the convergence rate. Combining the local training and global consensus, all agents converge to the optimum with only a single consensus communication at every training step.

On the one hand, the subdatasets of all agents are not required to be shared among neighbors or uploaded to a centralized node, which helps to preserve the sensitive and private data. On the other hand, this proposed distributed training framework is based on the decentralized communication topology with great flexibility and expansibility. This means that the convergence of the distributed neural networks is unaffected in the switching graphs and the situation that an agent quits or participates in this communication graph on the condition that it always remains a spanning tree [35]. The procedure for the distributed heuristic adaptive neural networks is detailed in Table I.

We take Fig. 1(b) as an example of the communication topology for distributed training, where the entire dataset is evenly partitioned and stored on six initialized neural networks (agents) over the graph. First, these agents are trained on their local subdatasets and share their model parameters among directly connected neighbors under the communication graph as shown in Fig. 2(a). After some iterations, we assume that the connection between the agent 6 and the communication graph is cut off. In this case, the agent 6 can only train with its own
TABLE I

Procedure of Distributed Heuristic Adaptive NEURAL NETWORKS

local subdataset but not communicate with other agents, while the other five agents form a new communication topology as shown in Fig. 2(b). Finally, the agent 6 joins in the graph again and the six agents form another communication topology as shown in Fig. 2(c). This changing process of the topologies in the simulation is to demonstrate that all agents still converge to the optimum in switching communication graph and the situation that any agent quits or participates in the communication graph on the condition that it always has a spanning tree.

In this article, four different algorithms are compared to validate the superiority of the distributed heuristic adaptive neural networks with variance reduction. These algorithms are tested on a large-scale dataset BlogFeedback [36] (downloaded from the University of California, Irvine (UCI) Machine Learning Repository) for the task of regression, which contains 52397 samples with 280 features. Besides, the neural networks on all agents have the same structure, with the sigmoid function being the activation function. All of the neural networks are randomly initialized in the uniform distribution $[-1,+1]$ with all inputs normalized in $[0,1]$. The following four algorithms are conducted in the above-mentioned switching graphs as shown in Fig. 2, with RMSE applied to assess their performance.

1) Distributed Heuristic Adaptive Training Using Batch Gradient: Six neural networks are trained locally using the batch gradient methods and the heuristic adaptive consensus algorithm is used to globally update their model parameters over the graph.

2) Distributed Training Using Batch Gradient: As before, the batch gradient descent is used to locally optimize the neural networks, while the consensus algorithm with a fixed weighted connectivity matrix is used to make all agents converge to each other.

3) Distributed Heuristic Adaptive Training Using SGD: In this case, SGD is used to optimize the neural networks, with the heuristic adaptive consensus algorithm globally updating their model parameters.

4) Distributed Heuristic Adaptive Training Using SVRG: Similar to before, the heuristic adaptive consensus algorithm is used for distributed training, with SVRG locally optimizing neural networks. 


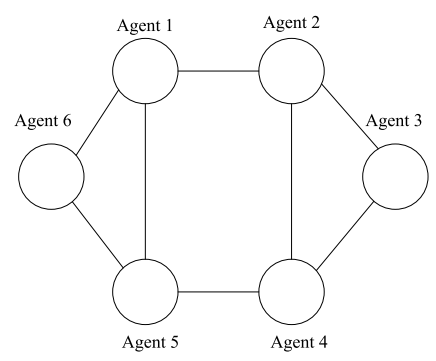

(a)

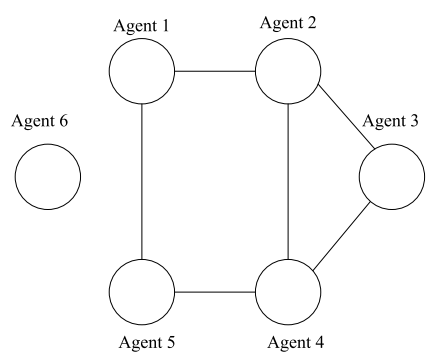

(b)

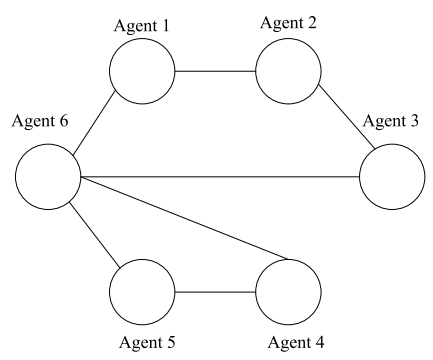

(c)

Fig. 2. Switching communication graphs for the simulations. (a) Initial communication graph. (b) Intermediate communication graph. (c) Final communication graph.

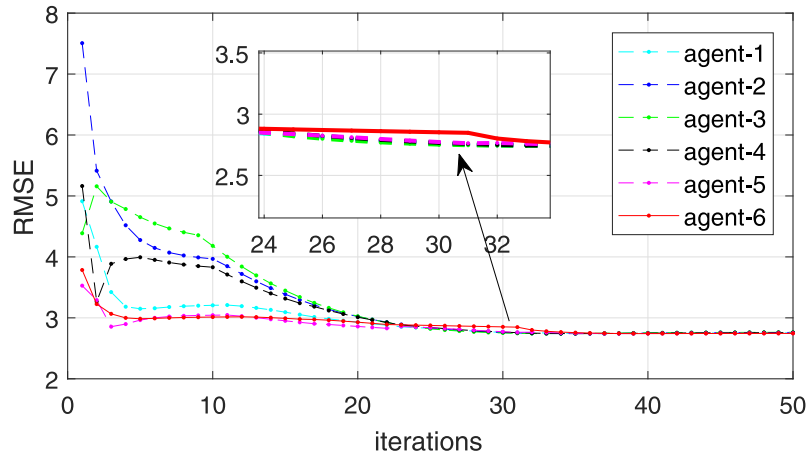

Fig. 3. Distributed heuristic adaptive training using batch gradient.

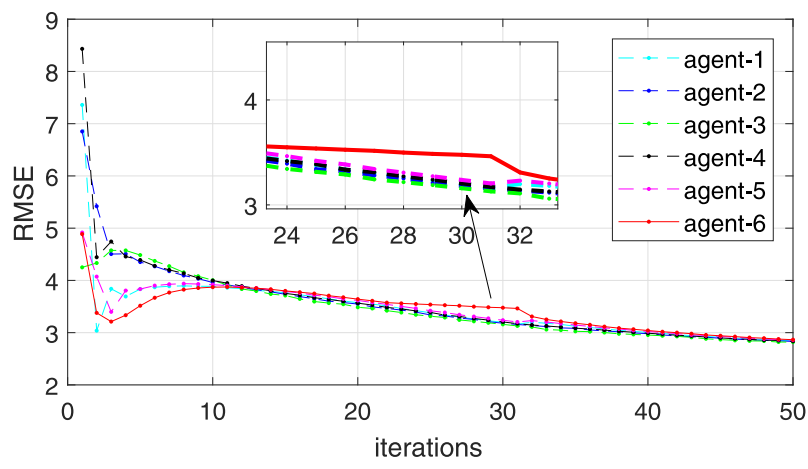

Fig. 4. Distributed training using batch gradient.

Figs. 3-6 are the simulation results on the above four algorithms, where we can find that the agents of all the four figures can approach the optimal model while converging to each other after enough iterations, despite the great difference in their initial statuses. Meanwhile, in all the figures except for Fig. 5, there is a clear gap between the agent 6 and the other agents from 21st to 31st iteration, when the agent 6 leaves the graph Fig. 2(b) and joins in again to form a new graph Fig. 2(c), respectively. More specifically, after the agent 6 leaves the communication graph at 21st iteration, the other five agents in Fig. 2(b) can still converge to the optimum in union, while the convergence speed of agent 6 slows down, comparing to other five agents. This means that the proposed distributed heuristic adaptive training method is more efficient and robust to the failure of an agent over the graph. Furthermore, after the agent 6 joins in and form another

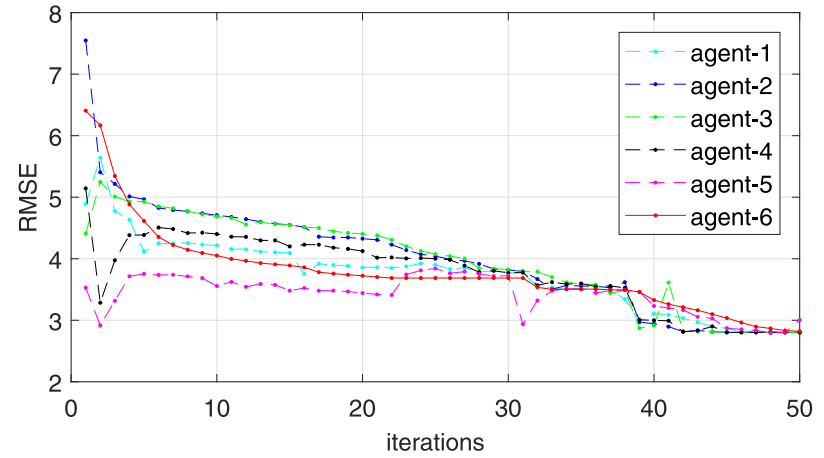

Fig. 5. Distributed heuristic adaptive training using SGD.

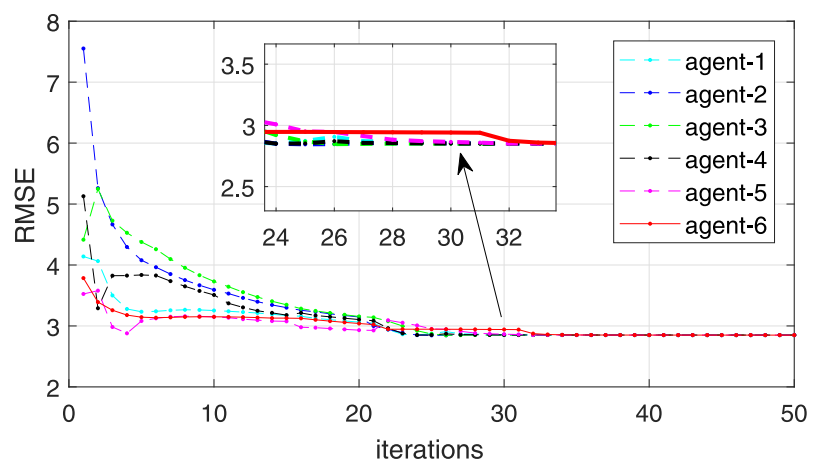

Fig. 6. Distributed heuristic adaptive training using SVRG.

communication graph at 31st iterations, all the six agents in Fig. 2(c) approach each other again and converge to the optimum in union. This demonstrates that the distributed training method is suitable for switching graphs with a spanning tree.

We can find from Fig. 3 that the heuristic adaptive consensus algorithm requires 20 and 30 iterations for all agents achieving consensus and converging to the optimal model, respectively. By comparison, as shown in Fig. 4, it is required 10 and 50 iterations for consensus algorithm with the fixed weighted connectivity matrix to reach consensus and the optimum, respectively. Although more iterations are needed to achieve consensus when it is compared with the consensus algorithm, the heuristic adaptive consensus algorithm requires much fewer iterations to reach the optimal model, which means that this method is more efficient and suitable for distributed training neural networks. 
Fig. 5 is the performance of distributed training using SGD, where we cannot find the gap shown in other figures, because the agents have not converged between 21 st and 31 st iterations. Besides, we can find that there are more fluctuations in Fig. 5 than in Fig. 3, which is harmful to the convergence. Therefore, a smaller learning rate should be taken for SGD to ensure convergence, which would further impair the convergence rate. As shown in Fig. 6, the simulation result of distributed training with SVRG exhibits nearly the same performance as the batch gradient (Fig. 3), which is more smoothly and shows fewer fluctuations, compared with SGD (Fig. 5). This verifies the effectiveness of the SVRG method, which shows better performance with only a little extra computational cost. What deserves to be mentioned is that a larger learning rate can be taken for SVRG because of the reduction of the variance introduced by stochastic gradient, which would further improve their performance and convergence rate. Here, the same learning rate was used in the simulations for all of the four algorithms for the convenience of comparisons.

\section{CONCLUSION}

In recent years, distributed training has become a hot research topic for its excellent ability in the machine learning problems on massive and privacy-related data. Besides, it is common nowadays that data samples are not available to be centralized or shared among agents because of computational limitations or privacy issues. However, it is still expected that the model is built based on the entire dataset rather than only learns from its local subdataset. For this problem, a distributed training method is designed based on the SVRG and the heuristic adaptive consensus algorithm for neural networks connected in switching communication graphs.

Through the theoretical analysis, we show that the SVRG reduces the variance introduced by SGD and improve its convergence rate with only a little extra computational cost. For distributed neural networks, it is proved that the consensusbased distributed training with sufficient consensus communication obtains the identical performance as the model built on the entire dataset. However, this method is computationally expensive and too straightforward as all agents are considered equally during the consensus process. Furthermore, the heuristic adaptive consensus algorithm is proposed to adaptively adjust the weighted connectivity matrix, where a better agent is more likely to have a larger influence on its neighbors. Convergence analysis demonstrates that all agents in switching graphs can still converge to the optimum with a single consensus communication at every training step, which saves plenty of communication costs. Finally, simulations give the results that the heuristic adaptive consensus algorithm requires fewer iterations than the consensus algorithm with the fixed weighted connectivity matrix for all agents to reach the optimum, and SVRG greatly decreases the fluctuation caused by SGD and improve its performance.

\section{REFERENCES}

[1] D. Kumar, J. C. Bezdek, M. Palaniswami, S. Rajasegarar, C. Leckie, and T. C. Havens, "A hybrid approach to clustering in big data," IEEE Trans. Cybern., vol. 46, no. 10, pp. 2372-2385, Oct. 2016.
[2] M. Mao, J. Lu, G. Zhang, and J. Zhang, "Multirelational social recommendations via multigraph ranking," IEEE Trans. Cybern., vol. 47, no. 12, pp. 4049-4061, Dec. 2017.

[3] W. Kim, M. S. Stanković, K. H. Johansson, and H. J. Kim, "A distributed support vector machine learning over wireless sensor networks," IEEE Trans. Cybern., vol. 45, no. 11, pp. 2599-2611, Nov. 2015.

[4] A. Broggi, A. Zelinsky, Ü. Özgüner, and C. Laugier, "Intelligent vehicles," in Springer Handbook of Robotics. Heidelberg, Germany: Springer, 2016, pp. 1627-1656.

[5] R. Gravina, P. Alinia, H. Ghasemzadeh, and G. Fortino, "Multisensor fusion in body sensor networks: State-of-the-art and research challenges," Inf. Fusion, vol. 35, pp. 68-80, May 2017.

[6] N. Shaker, S. Asteriadis, G. N. Yannakakis, and K. Karpouzis, "Fusing visual and behavioral cues for modeling user experience in games," IEEE Trans. Cybern., vol. 43, no. 6, pp. 1519-1531, Dec. 2013.

[7] L. Lin, W. Yang, C. Li, J. Tang, and X. Cao, "Inference with collaborative model for interactive tumor segmentation in medical image sequences," IEEE Trans. Cybern., vol. 46, no. 12, pp. 2796-2809, Dec. 2016.

[8] B. Lei, P. Yang, T. Wang, S. Chen, and D. Ni, "Relational-regularized discriminative sparse learning for alzheimer's disease diagnosis," IEEE Trans. Cybern., vol. 47, no. 4, pp. 1102-1113, Apr. 2017.

[9] S. Boyd, N. Parikh, E. Chu, B. Peleato, J. Eckstein, "Distributed optimization and statistical learning via the alternating direction method of multipliers," Found. Trends Mach. Learn., vol. 3, no. 1, pp. 1-122, 2011.

[10] T.-H. Chang, M. Hong, W.-C. Liao, and X. Wang, "Asynchronous distributed ADMM for large-scale optimization-Part I: Algorithm and convergence analysis," IEEE Trans. Signal Process., vol. 64, no. 12, pp. 3118-3130, Jun. 2016.

[11] P. A. Forero, A. Cano, and G. B. Giannakis, "Consensus-based distributed support vector machines," J. Mach. Learn. Res., vol. 11, pp. 1663-1707, May 2010.

[12] X. Miao, Y. Liu, H. Zhao, and C. Li, "Distributed online one-class support vector machine for anomaly detection over networks," IEEE Trans. Cybern., to be published.

[13] H. P. Graf, E. Cosatto, L. Bottou, I. Dourdanovic, and V. Vapnik, "Parallel support vector machines: The cascade SVM," in Proc. Adv. Neural Inf. Process. Syst., 2005, pp. 521-528.

[14] J.-P. Zhang, Z.-W. Li, and J. Yang, "A parallel SVM training algorithm on large-scale classification problems," in Proc. 4th Int. Conf. Mach. Learn. Cybern., Aug. 2005, pp. 1637-1641.

[15] S. Scardapane, D. Wang, M. Panella, and A. Uncini, "Distributed learning for random vector functional-link networks," Inf. Sci., vol. 301, pp. 271-284, Apr. 2015.

[16] J. Chen, B. Chen, Z. Zeng, and P. Jiang, "Event-triggered synchronization strategy for multiple neural networks with time delay," IEEE Trans. Cybern., to be published, doi: 10.1109/TCYB.2019.2911029.

[17] J.-L. Wang, X.-X. Zhang, H.-N. Wu, T. Huang, and Q. Wang, "Finite-time passivity of adaptive coupled neural networks with undirected and directed topologies," IEEE Trans. Cybern., to be published, doi: 10.1109/TCYB.2018.2882252.

[18] L. Ma and W. Bian, "A novel multiagent neurodynamic approach to constrained distributed convex optimization," IEEE Trans. Cybern., to be published, doi: 10.1109/TCYB.2019.2895885.

[19] R. Olfati-Saber, J. A. Fax, and R. M. Murray, "Consensus and cooperation in networked multi-agent systems," Proc. IEEE, vol. 95, no. 1, pp. 215-233, Jan. 2007.

[20] L. Georgopoulos and M. Hasler, "Distributed machine learning in networks by consensus," Neurocomputing, vol. 124, pp. 2-12, Jan. 2014.

[21] B. Liu, Z. Ding, and C. Lv, "Distributed training for multi-layer neural networks by consensus," IEEE Trans. Neural Netw. Learn. Syst., to be published, doi: 10.1109/TNNLS.2019.2921926.

[22] X. Lian, C. Zhang, H. Zhang, C.-J. Hsieh, W. Zhang, and J. Liu, "Can decentralized algorithms outperform centralized algorithms? a case study for decentralized parallel stochastic gradient descent," in Proc. Adv. Neural Inf. Process. Syst., Dec. 2017, pp. 5336-5346.

[23] S. Ruder. An Overview of Gradient Descent Optimization Algorithms. Accessed: Sep. 2016. [Online]. Available: arXiv:1609.04747

[24] L. Bottou, "Large-scale machine learning with stochastic gradient descent," in Proc. Int. Conf. Comput. Statist. (COMPSTAT). 2010, pp. 177-186.

[25] R. Johnson and T. Zhang, "Accelerating stochastic gradient descent using predictive variance reduction," in Proc. Adv. Neural Inf. Process. Syst., 2013, pp. 315-323. 
[26] M. Li et al., "Scaling distributed machine learning with the parameter server," in Proc. USENIX Conf. Oper. Syst. Design Implement. (OSDI), vol. 14, 2014, pp. 583-598.

[27] J. Konečnỳ, H. B. McMahan, F. X. Yu, P. Richtárik, A. T. Suresh, and D. Bacon. Federated Learning: Strategies for Improving Communication Efficiency. Accessed: Oct. 2016. [Online]. Available: arXiv:1610.05492

[28] R. Olfati-Saber and R. M. Murray, "Consensus problems in networks of agents with switching topology and time-delays," IEEE Trans. Autom. Control, vol. 49, no. 9, pp. 1520-1533, Sep. 2004.

[29] L. Xiao, S. Boyd, and S.-J. Kim, "Distributed average consensus with least-mean-square deviation," J. Parallel Distrib. Comput., vol. 67, no. 1, pp. 33-46, 2007.

[30] F. L. Lewis, H. Zhang, K. Hengster-Movric, and A. Das, Cooperative Control of Multi-Agent Systems: Optimal and Adaptive Design Approaches. London, U.K.: Springer, 2013.

[31] Y.-J. Gong et al., "Genetic learning particle swarm optimization," IEEE Trans. Cybern., vol. 46, no. 10, pp. 2277-2290, Oct. 2016.

[32] X. Zeng, W. Wang, C. Chen, and G. G. Yen, "A consensus community-based particle swarm optimization for dynamic community detection," IEEE Trans. Cybern., to be published, doi: 10.1109/TCYB.2019.2938895.

[33] G. Lan, S. Lee, and Y. Zhou. Communication-Efficient Algorithms for Decentralized and Stochastic Optimization. Accessed: Jan. 2017. [Online]. Available: arXiv: 1701.03961

[34] B. Sirb and X. Ye, "Consensus optimization with delayed and stochastic gradients on decentralized networks," in Proc. IEEE Int. Conf. Big Data (Big Data), Dec. 2016, pp. 76-85.

[35] H. Jiang, Q. Bi, and S. Zheng, "Impulsive consensus in directed networks of identical nonlinear oscillators with switching topologies," Commun. Nonlinear Sci. Numer. Simulat., vol. 17, no. 1, pp. 378-387, 2012.

[36] K. Buza, "Feedback prediction for blogs," in Data Analysis, Machine Learning and Knowledge Discovery. Basel, Switzerland: Springer, 2014, pp. $145-152$.

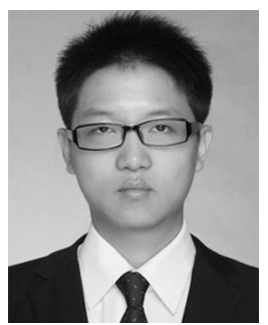

Bo Liu received the B.Eng. and M.Eng. degrees from the Wuhan University of Technology, Wuhan, China, in 2014 and 2017, respectively. He is currently pursing the Ph.D. degree in electrical and electronic engineering with the University of Manchester, Manchester, U.K.

His current research interests include distributed optimization, distributed machine learning, and their applications.

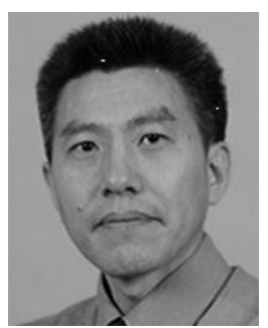

Zhengtao Ding (SM'03) received the B.Eng. degree from Tsinghua University, Beijing, China, in 1984, and the M.Sc. degree in systems and control and Ph.D. degree in control systems from the University of Manchester Institute of Science and Technology, Manchester, U.K., in 1986 and 1989, respectively.

After working as a Lecturer with Ngee Ann Polytechnic, Singapore, for ten years, in 2003, he joined the University of Manchester, Manchester, where he is a Professor of Control Systems with the Department of Electrical and Electronic Engineering. He has authored the book Nonlinear and Adaptive Control Systems (IET, 2013) and a number of journal papers. His research interest includes nonlinear and adaptive control theory and their applications.

Prof. Ding has served as an Associate Editor for the IEEE TRANSACTIONS on Automatic Control, Transactions of the Institute of Measurement and Control, Control Theory and Technology, Mathematical Problems in Engineering, Unmanned Systems, and the International Journal of Automation and Computing. 